\title{
ARTICLE
}

Clinical Study

\section{A first-in-man phase 1 study of the DNA-dependent protein kinase inhibitor peposertib (formerly M3814) in patients with advanced solid tumours}

\author{
Mark T. J. van Bussel (iD) ${ }^{1}$, Ahmad Awada ${ }^{2}$, Maja J. A. de Jonge ${ }^{3}$, Morten Mau-Sørensen (iD) ${ }^{4}$, Dorte Nielsen ${ }^{5}$, Patrick Schöffski ${ }^{6}$, \\ Henk M. W. Verheul ${ }^{7}$, Barbara Sarholz ${ }^{8}$, Karin Berghoff ${ }^{8}$, Samer El Bawab ${ }^{8}$, Mirjam Kuipers ${ }^{8}$, Lars Damstrup ${ }^{8,10}$, Ivan Diaz-Padilla ${ }^{9}$ and \\ Jan H. M. Schellens ${ }^{1,11}$
}

BACKGROUND: This open-label, phase 1 trial (NCT02316197) aimed to determine the maximum-tolerated dose (MTD) and/or recommended phase 2 dose (RP2D) of peposertib (formerly M3814), a DNA-dependent protein kinase (DNA-PK) inhibitor in patients with advanced solid tumours. Secondary/exploratory objectives included safety/tolerability, pharmacokinetic/pharmacodynamic profiles and clinical activity.

METHODS: Adult patients with advanced solid tumours received peposertib $100-200 \mathrm{mg}$ once daily or $150-400 \mathrm{mg}$ twice daily (BID) in 21-day cycles.

RESULTS: Thirty-one patients were included (median age 66 years, $61 \%$ male). One dose-limiting toxicity, consisting of mainly gastrointestinal, non-serious adverse events (AEs) and long recovery duration, was reported at 300 mg BID. The most common peposertib-related AEs were nausea, vomiting, fatigue and pyrexia. The most common peposertib-related Grade 3 AEs were maculopapular rash and nausea. Peposertib was quickly absorbed systemically (median $T_{\max } 1.1-2.5 \mathrm{~h}$ ). The p-DNA-PK/t-DNA-PK ratio decreased consistently in peripheral blood mononuclear cells $3-6 \mathrm{~h}$ after doses $\geq 100 \mathrm{mg}$. The best overall response was stable disease (12 patients), lasting for $\geq 12$ weeks in seven patients.

CONCLUSIONS: Peposertib was well-tolerated and demonstrated modest efficacy in unselected tumours. The MTD was not reached; the RP2D was declared as $400 \mathrm{mg}$ BID. Further studies, mainly with peposertib/chemo-radiation, are ongoing.

CLINICAL TRIAL REGISTRATION: NCT02316197

British Journal of Cancer (2021) 124:728-735; https://doi.org/10.1038/s41416-020-01151-6

\section{BACKGROUND}

DNA double-strand breaks (DSBs) are the most cytotoxic type of DNA lesions, which if left unrepaired, can cause cell-cycle arrest via checkpoint activation and subsequent cell death. ${ }^{1}$ Complex signalling pathways, collectively termed the DNA damage response (DDR), exist within cells to detect and repair DNA damage, including DNA DSBs. ${ }^{1}$ Defects in DDR pathways can lead to the accumulation of non-lethal DNA damage and genomic instability, which is a hallmark of many cancers and drives tumourigenesis. ${ }^{2}$

Many anti-cancer treatments, such as radiotherapy and some chemotherapies, work by inducing DNA DSBs in tumour cells. The efficacy of such treatments can be compromised by the efficient repair of DNA damage through activation of the DDR, including DNA-dependent protein kinase (DNA-PK). ${ }^{3}$ DNA-PK is a serine/ threonine protein kinase that plays a critical role in the DDR and regulates DNA DSB repair via the non-homologous end joining (NHEJ) pathway. ${ }^{4,5}$

Peposertib (formerly M3814) is an orally administered, smallmolecule, selective DNA-PK inhibitor that blocks DNA-PK kinase activity at sub-nanomolar concentrations, inhibiting its ability to function in the DNA repair process leading to the persistence of DNA DSBs and subsequent cell death. ${ }^{6-8}$ Whilst peposertib has previously demonstrated selectivity for several members of the phosphoinositide 3 kinase (PI3K) and PI3K-related family of proteins, of which DNA-PK, ataxia-telangiectasia-mutated (ATM) and ataxia-telangiectasia and Rad3-related (ATR) kinases are members, its potency against DNA-PK was substantially greater than against other family members. ${ }^{8}$ Additionally, peposertib demonstrated synergy with radiotherapy and DSB-inducing chemotherapies (e.g. etoposide, doxorubicin) in preclinical studies

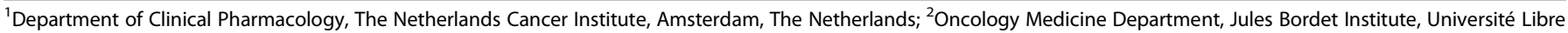
de Bruxelles, Brussels, Belgium; ${ }^{3}$ Medical Oncology, Erasmus MC Cancer Institute, Rotterdam, The Netherlands; ${ }^{4}$ Department of Oncology, Rigshospitalet, Copenhagen, Denmark;

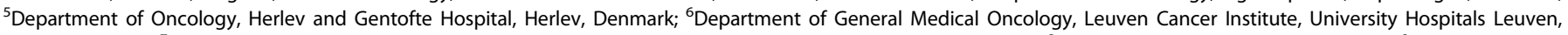

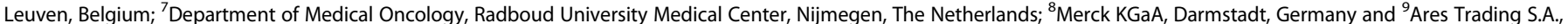
Eysins, Switzerland; an Affiliate of Merck KGaA, Darmstadt, Germany Correspondence: Mark T. J. van Bussel (m.v.bussel@nki.nl)

${ }^{10}$ Present address: Debiopharm International S.A., Lausanne, Switzerland

${ }^{11}$ Present address: Synthon Biopharmaceuticals, Nijmegen, The Netherlands
}

Received: 18 June 2020 Revised: 7 October 2020 Accepted: 22 October 2020

Published online: 24 November 2020 
by preventing the repair of radiation- or chemotherapy-induced DNA DSBs via the NHEJ pathway. ${ }^{6-10}$

The purpose of this first-in-man, open-label, phase 1 trial (NCT02316197) was to determine the maximum-tolerated dose (MTD) and/or the recommended phase 2 dose (RP2D) of peposertib. To our knowledge, this is the first full report of an oral DNA-PK inhibitor in humans. The results of this study have been partially presented at the American Society for Clinical Oncology Annual Meeting in $2017^{11}$ and at the European Society for Medical Oncology Annual Meeting in 2018. ${ }^{12}$

\section{METHODS}

Trial design

This phase 1, first-in-man, open-label, dose-escalation study (NCT02316197) was designed to explore the safety, tolerability, pharmacokinetic (PK) and pharmacodynamic (PD) profiles, and clinical activity of peposertib administered daily as a single agent to patients with advanced solid tumours likely to have alterations in DNA repair mechanisms. Following screening and baseline evaluations, patients received peposertib until disease progression, unacceptable toxicity, withdrawal of consent or other reasons necessitating withdrawal (Supplementary Fig. S1).

Dose escalation was by use of a standard ' $3+3$ ' design, based on the presence/absence of dose-limiting toxicities (DLTs). Patients in the first dose cohort received peposertib continuously in 21-day cycles at a starting dose of $100 \mathrm{mg}$ once daily (QD). The starting dose was considered to have acceptable safety and to be in the range of the biologically active dose based on in vivo pharmacology data. ${ }^{7}$ Patients in the second cohort received peposertib at a dose of $200 \mathrm{mg}$ QD. Subsequent cohorts received ascending doses of peposertib as follows: $150 \mathrm{mg}$ twice daily (BID); $200 \mathrm{mg} \mathrm{BID;} 300 \mathrm{mg}$ BID; and $400 \mathrm{mg}$ BID.

The study was conducted in accordance with the ethical principles of the International Council for Harmonisation guideline for Good Clinical Practice and the Declaration of Helsinki, as well as with applicable local regulations.

\section{Patients}

Patients aged $\geq 18$ years with advanced solid tumours, for whom no other standard surgical, radiation or systemic anti-cancer therapies were available, and with tumour accessible for biopsies, and measurable or evaluable disease by Response Evaluation Criteria in Solid Tumours (RECIST) v1.1 ${ }^{13}$ were eligible for inclusion in the study. Although not mandatory, the intention was to select patients based on the likelihood of alterations in DNA repair mechanisms (triple-negative breast, serous epithelial ovary, bladder, microsatellite instability-high colon, lung, castration-resistant prostate, stomach or uterine cancer). Patients were excluded from the study if they had an Eastern Cooperative Oncology Group performance status (ECOG PS) $>1$; had received any anti-cancer therapy (except for luteinising hormone-releasing hormone analogues) or any other investigational agent within 28 days of the first dose of peposertib; had received extensive radiotherapy on $>30 \%$ of bone marrow reserves or prior bone marrow/stem cell transplantation within 5 years of study start; or were receiving medications/herbal supplements known to be potent inhibitors or inducers of CYP3A or CYP2C19. All patients provided written informed consent.

\section{Study objectives and endpoints}

The primary objective was to determine the MTD and/or the RP2D of peposertib assessed as the proportion of patients who experienced at least one DLT during the first 21-day treatment cycle. The MTD was defined as the dose level below the dose at which two out of six patients experienced a DLT. Once the MTD was established, the RP2D was to be defined by the Safety Monitoring Committee (SMC), either at the MTD level or below, depending on the available safety, efficacy, PK and PD data.
A DLT was defined as any of the following toxicities considered to be possibly related to peposertib by the sponsor and/or investigator during Cycle 1: a treatment-emergent adverse event (TEAE) of potential clinical significance such that further dose escalation would expose patients to unacceptable risk; evidence of possible treatment-related hepatocellular injury for $\geq 3$ days; any Grade 4 liver enzyme elevation; any Grade $\geq 3$ toxicity (defined according to National Cancer Institute Common Terminology Criteria for Adverse Events v4.03, ${ }^{14}$ and excluding diarrhoea, nausea and vomiting of $<3$ days duration following adequate therapy, Grade 3 skin toxicity resolving to Grade $\leq 2$ with supporting measures within 7 days, fatigue or headache of $<7$ days duration following initiation of adequate supportive care, any other single laboratory value out of the normal range that was not correlated to clinically significant symptoms, Grade 3 thrombocytopenia without bleeding, neutropenia lasting for $\leq 5$ days and not associated with fever); any toxicity related to study drug resulting in $\geq 20 \%$ of the planned dose to be missed in Cycle 1. In addition, a DLT could be identified by the SMC as any TEAE that impaired daily function or abnormality occurring in patients treated with peposertib at any time during the dose escalation part of the study. TEAEs were defined as adverse events (AEs) observed from the first dose of peposertib to 30 days after the last dose, whilst a serious TEAE was defined as any TEAE that was life threatening, required hospitalisation or prolongation of existing hospitalisation, resulted in death, or was otherwise considered as medically important.

Secondary objectives included evaluation of the safety and tolerability of peposertib, assessment of PK and exploration of antitumour activity. Safety was assessed through the recording, reporting and analysis of baseline medical conditions, AEs, physical examination findings (including vital signs and monitoring for bleeding), laboratory tests, ECOG PS and 12-lead electrocardiograms. Based on preclinical data showing reversible inhibition of platelet aggregation at micromolar concentrations, the potential impact of peposertib on human cyclooxygenase 1 was investigated by evaluating the inhibition of platelet aggregation in six aspirinnaïve patients treated at the RP2D. PK was assessed using blood samples collected pre-treatment and on-treatment. The following PK parameters were evaluated: maximum observed concentration $\left(C_{\max }\right)$; dose-normalised $C_{\max }\left(C_{\max } /\right.$ dose $)$; time to $C_{\max }\left(t_{\max }\right)$; area under the plasma concentration-time curve (AUC) from 0 to $12 \mathrm{~h}$ $\left(A C_{0-12}\right)$; dose-normalised $A C_{0-12}\left(A_{U} C_{0-12} /\right.$ dose); $A U C$ from 0 to infinity $\left(A \cup C_{0-\infty} ;\right.$ day 1 only); apparent elimination half-life; apparent total body clearance of drug; apparent total body clearance at steady state of drug (cycle 2 only); apparent volume of distribution during terminal phase $\left(V_{\mathrm{z} / \mathrm{f}}\right)$; accumulation ratio for AUC $\left(R_{\mathrm{acc}(\mathrm{AUC})}\right)$; and accumulation ratio for $C_{\max }\left(R_{\text {acc }(\text { (max) }}\right)$. Tumour response was evaluated according to RECIST ( $v 1.1$ ). Target and non-target lesions were measured by computed tomography or magnetic resonance imaging every 6 weeks.

Exploratory objectives included assessing the PD in peripheral blood mononuclear cells (PBMCs), isolated from serial blood samples collected pre-treatment and on-treatment. PBMCs were exposed to the DNA-damaging agent bleomycin to induce DNAPK activity ex vivo. The level of the autophosphorylated form of DNA-PK on Ser $^{2056}$ (p-DNA-PK) was assessed as a PD biomarker for peposertib. Phospho-DNA-PK levels normalised to total DNA-PK ( $t-$ DNA-PK) were measured using the Erenna ${ }^{\circledR}$ Immunoassay System (Singulux, Alameda, California, USA) in PBMC lysates, and calculated as the p-DNA-PK concentration $(\mathrm{ng} / \mathrm{mL}) / \mathrm{t}-\mathrm{DNA}$-PK concentration $(\mathrm{ng} / \mathrm{mL})$. DNA-PK inhibition by peposertib ontreatment was expressed as the percentage change of the PD biomarker versus the pre-treatment value.

\section{Analyses}

The cut-off for the final analysis was when the last patient completed the dose-escalation phase (29 June 2017). The All Patients Analysis Set comprised all patients who provided informed 


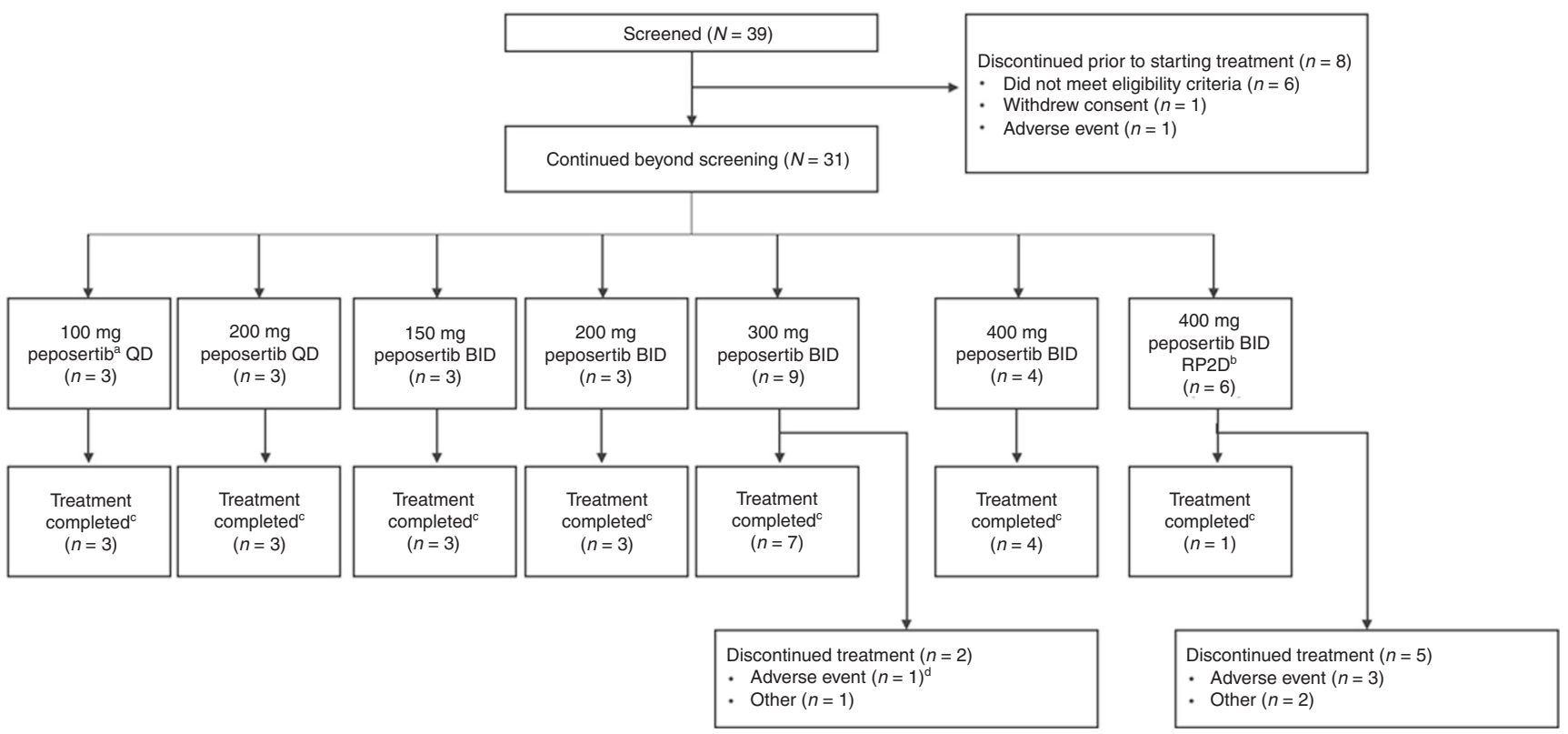

Fig. 1 Patient disposition. ${ }^{\mathrm{a}}$ Formerly M3814; ${ }^{\mathrm{b}}$ The six patients in the RP2D cohort were aspirin-naïve; ${ }^{\mathrm{C}}$ Patients were treated until disease progression or death; ${ }^{d}$ DLT, due to a combination of low grade, mainly gastrointestinal, non-serious AEs and the long duration of recovery following treatment discontinuation. BID twice daily, DLT dose-limiting toxicity, QD once daily, RP2D recommended phase 2 dose.

consent (screening failures plus enrolled patients) and was used to describe patient disposition and deaths. The Safety Analysis Set included patients who received at least one dose of peposertib and was used for all baseline and safety (except for DLTs) summaries. The Efficacy Analysis Set, including patients who received at least one treatment dose, was used for all efficacy summaries. The DoseEscalation Analysis Set included patients treated in dose-escalation cohorts who received at least $80 \%$ of peposertib planned doses in the first treatment cycle or who experienced a DLT during the first treatment cycle regardless of the amount of drug received (used for DLT summary). The PK Analysis Set (cycles 1 and 2) included patients who received at least the first dose of peposertib and provided PK samples as per protocol for at least $24 \mathrm{~h}$ following the first dose on day 1 of cycle 1 for the QD regimen, or for at least $12 \mathrm{~h}$ following first dose on day 1 of cycle 1 for the BID regimen. The Biomarker/ Pharmacogenomics Analysis Set included patients who received at least the first dose of study drug and provided at least one pre-dose sample and one post-dose sample.

TEAEs were summarised according to Medical Dictionary for Regulatory Activities (v20.0) preferred term and system organ class. All statistical analyses (except for PK) were performed using SAS 9.1.3 or higher. Tumour assessments, based on investigator evaluations of target, non-target and new lesions according to RECIST (v1.1), were used to derive the best overall response. Trial data were summarised by dose level.

\section{RESULTS}

Patient baseline demographics and disease characteristics Patient disposition is shown in Fig. 1. Of the 39 patients who were screened, eight patients did not receive peposertib: six did not meet the eligibility criteria, one withdrew consent and one did not continue beyond screening due to a serious AE (intestinal obstruction). Thirty-one patients were then included in the study (100 mg QD, $n=3 ; 200 \mathrm{mg} \mathrm{QD,} n=3 ; 150 \mathrm{mg} \mathrm{BID,} n=3 ; 200 \mathrm{mg}$ $\mathrm{BID}, n=3 ; 300 \mathrm{mg}$ BID, $n=9 ; 400 \mathrm{mg}$ BID, $n=4 ; 400 \mathrm{mg}$ BID [declared as the RP2D], aspirin-naïve, $n=6$ ). The numbers of patients included in each of the analysis sets were as follows: Safety/Efficacy Analysis Set, $n=31$; Dose-Escalation Analysis Set, $n=21$; PK Analysis Set, $n=31$; Biomarker Analysis Set, $n=31$. The
Table 1. Baseline patient demographics and disease characteristics.

\begin{tabular}{llr}
\hline Parameter & Categories/characteristics & Total $^{\mathrm{a}}$ \\
\hline Sex, $n$ (\%) & Male & $19(61)$ \\
& Female & $12(39)$ \\
Race, $n$ (\%) & Caucasian & $27(87)$ \\
& Other & $4(13)$ \\
Age (years) & Mean (SD) & $62(12)$ \\
& Median (range) & $66(25-78)$ \\
ECOG PS, $n$ (\%) & 0 & $10(32)$ \\
& 1 & $21(68)$ \\
Primary tumour location & Colon/rectum (colorectal) & $9(29)$ \\
(tumour type added where & Liver (hepatocarcinoma) & $2(7)$ \\
applicable), $n$ (\%) & Skin (malignant melanoma) & $2(7)$ \\
& Lung (NSCLC) & $2(7)$ \\
& Bone (osteosarcoma) & $2(7)$ \\
& Parotid gland & $2(7)$ \\
& Head/neck (SCCHN) & $2(7)$ \\
& Adrenal & $1(3)$ \\
& Bladder & $1(3)$ \\
& Breast & $1(3)$ \\
& Bile duct & $1(3)$ \\
& (cholangiocarcinoma) & $1(3)$ \\
& Thigh (liposarcoma) & $1(3)$ \\
& Ovary & $1(3)$ \\
& Pancreas & $1(3)$ \\
& Kidney (renal cell cancer) & $1(3)$ \\
& Uterus & $1(3)$ \\
\hline & Other & \\
& Oncology & \\
& & \\
& & \\
& &
\end{tabular}

ECOG PS Eastern Cooperative Oncology Group performance status, NSCLC non-small cell lung cancer, SCCHN squamous cell carcinoma of the head and neck, SD standard deviation.

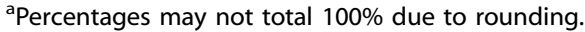

${ }^{\mathrm{b}} \mathrm{Skin}$ or other parts of the face.

first patient's first visit was on 14 January 2015 and the last patient's last visit was on 29 June 2017.

Patient baseline characteristics are presented in Table 1. The median (range) age was 66 (25-78) years; 19/31 (61\%) patients 


\begin{tabular}{|c|c|c|c|c|c|c|c|c|c|}
\hline Preferred term $n$ (\%) & Grade $^{\mathrm{b}}$ & $\begin{array}{l}100 / \mathrm{QD} \\
n=3\end{array}$ & $\begin{array}{l}200 / \mathrm{QD} \\
n=3\end{array}$ & $\begin{array}{l}150 / \mathrm{BID} \\
n=3\end{array}$ & $\begin{array}{l}200 / \mathrm{BID} \\
n=3\end{array}$ & $\begin{array}{l}300 / \mathrm{BID} \\
n=9\end{array}$ & $\begin{array}{l}400 / \mathrm{BID} \\
n=4\end{array}$ & $\begin{array}{l}\text { 400/BID (RP2D) } \\
n=6\end{array}$ & $\begin{array}{l}\text { Total } \\
N=31\end{array}$ \\
\hline Patients with $\geq 1$ event & & $1(33)$ & $2(67)$ & $2(67)$ & $0(0)$ & $8(89)$ & $3(75)$ & $6(100)$ & $22(71)$ \\
\hline Nausea & Any & $0(0)$ & $0(0)$ & $0(0)$ & $0(0)$ & $2(22)$ & $2(50)$ & $4(67)$ & $8(26)$ \\
\hline & Grade 3 & $0(0)$ & $0(0)$ & $0(0)$ & $0(0)$ & $0(0)$ & $0(0)$ & $0(0)$ & $0(0)$ \\
\hline \multirow[t]{2}{*}{ Fatigue } & Any & $0(0)$ & $0(0)$ & $1(33)$ & $0(0)$ & $5(56)$ & $0(0)$ & $0(0)$ & $6(19)$ \\
\hline & Grade 3 & $0(0)$ & $0(0)$ & $0(0)$ & $0(0)$ & $1(11)$ & $0(0)$ & $0(0)$ & $1(3)$ \\
\hline \multirow[t]{2}{*}{ Pyrexia } & Any & $0(0)$ & $0(0)$ & $0(0)$ & $0(0)$ & $1(11)$ & $1(25)$ & $3(50)$ & $5(16)$ \\
\hline & Grade 3 & $0(0)$ & $0(0)$ & $0(0)$ & $0(0)$ & $0(0)$ & $1(25)$ & $0(0)$ & $1(3)$ \\
\hline Maculopapular rash & Grade 3 & $0(0)$ & $0(0)$ & $0(0)$ & $0(0)$ & $1(11)$ & $1(25)$ & $2(33)$ & $4(13)$ \\
\hline \multirow[t]{2}{*}{ Constipation } & Any & $0(0)$ & $1(33)$ & $0(0)$ & $0(0)$ & $2(22)$ & $0(0)$ & $0(0)$ & $3(10)$ \\
\hline & Grade 3 & $0(0)$ & $0(0)$ & $0(0)$ & $0(0)$ & $0(0)$ & $0(0)$ & $0(0)$ & $0(0)$ \\
\hline \multirow[t]{2}{*}{ Diarrhoea } & Any & $0(0)$ & $1(33)$ & $1(33)$ & $0(0)$ & $0(0)$ & $0(0)$ & $1(17)$ & $3(10)$ \\
\hline & Grade 3 & $0(0)$ & $0(0)$ & $0(0)$ & $0(0)$ & $0(0)$ & $0(0)$ & $0(0)$ & $0(0)$ \\
\hline \multirow[t]{2}{*}{ Rash } & Any & $0(0)$ & $0(0)$ & $0(0)$ & $0(0)$ & $2(22)$ & $0(0)$ & $1(17)$ & $3(10)$ \\
\hline & Grade 3 & $0(0)$ & $0(0)$ & $0(0)$ & $0(0)$ & $1(11)$ & $0(0)$ & $1(17)$ & $2(7)$ \\
\hline \multirow[t]{2}{*}{ Periorbital oedema } & Any & $0(0)$ & $0(0)$ & $0(0)$ & $0(0)$ & $1(11)$ & $0(0)$ & $1(17)$ & $2(7)$ \\
\hline & Grade 3 & $0(0)$ & $0(0)$ & $0(0)$ & $0(0)$ & $0(0)$ & $0(0)$ & $0(0)$ & $0(0)$ \\
\hline \multirow[t]{2}{*}{ Dry mouth } & Any & $0(0)$ & $0(0)$ & $0(0)$ & $0(0)$ & $2(22)$ & $0(0)$ & $0(0)$ & $2(7)$ \\
\hline & Grade 3 & $0(0)$ & $0(0)$ & $0(0)$ & $0(0)$ & $0(0)$ & $0(0)$ & $0(0)$ & $0(0)$ \\
\hline
\end{tabular}

were male; $27 / 31$ (87\%) were Caucasian; ECOG PS at baseline was 0 in ten (32\%) patients and 1 in $68 \%$ of patients.

The majority of patients were treated until disease progression or death, except for two patients in the $300 \mathrm{mg}$ BID cohort (one discontinued due to an AE [DLT, due to a combination of lowgrade, mainly gastrointestinal, non-serious $A E s$ and the long duration of recovery following treatment discontinuation] and the other due to patient request after experiencing fatigue (Grade 3) amongst other Grade $\leq 2 \mathrm{AEs}$ ), and five patients in the $400 \mathrm{mg}$ BID (RP2D) cohort who discontinued due to AEs ( $n=3$; colitis in one patient [unrelated to peposertib]; maculopapular rash in one patient [related to peposertib]; nausea and papular rash in one patient [both related to peposertib]; all were considered serious and all were Grade 3$)$ and clinical progressive disease $(n=2)$.

Dose-limiting toxicities and maximum-tolerated dose

Safety evaluation was based on peposertib exposure of up to a maximum of 484 days (69 weeks). One DLT was reported for one patient in the $300 \mathrm{mg}$ BID cohort: no individual $A E$ qualified as a DLT; however, a combination of non-serious AEs (stomatitis, decreased appetite, dysgeusia, erythema, urticaria, fatigue and nausea) and long recovery duration following treatment discontinuation was considered as a DLT. The MTD of peposertib was not reached at $400 \mathrm{mg}$ BID and dose escalation was stopped at 400 $\mathrm{mg}$ BID. As there were no DLTs reported at this dose level, $400 \mathrm{mg}$
BID was declared as an acceptable RP2D. This RP2D was further supported by the exposure levels observed at $400 \mathrm{mg}$ BID to be in the expected range of target engagement. ${ }^{8}$ Six aspirin-naïve patients (see above) were enrolled at the RP2D to confirm the safety and tolerability and to the effects of peposertib in platelet aggregation. The lack of objective responses or clearly identifiable efficacy signals in the patients treated precluded the initiation of the dose-expansion cohort of the study.

Safety

The median (range) treatment duration with peposertib was 6.0 (0.3-69.0) weeks. All 31 patients had at least one TEAE, and most $(71 \%)$ had at least one TEAE related to peposertib. Nausea $(n=$ $17)$, fatigue $(n=14)$, pyrexia $(n=11)$ and constipation $(n=10)$ were the most common TEAEs. Nausea $(n=8)$, vomiting $(n=6)$, fatigue $(n=6)$ and pyrexia $(n=5)$ were the most frequently reported TEAEs related to peposertib (Table 2). Twenty-one patients $(68 \%)$ had at least one TEAE of Grade $\geq 3$. Peposertibrelated Grade 3 TEAEs occurred in seven (23\%) patients. The most common Grade 3 peposertib-related TEAEs were maculo-papular rash $(n=4)$ and nausea $(n=2)$. There were no peposertib-related Grade 4 TEAEs.

Seventeen patients $(55 \%)$ had a serious TEAE, most of which were Grade $\leq 3$. However, two patients treated with peposertib $400 \mathrm{mg}$ BID reported Grade 4 TEAEs. Peposertib-related serious 


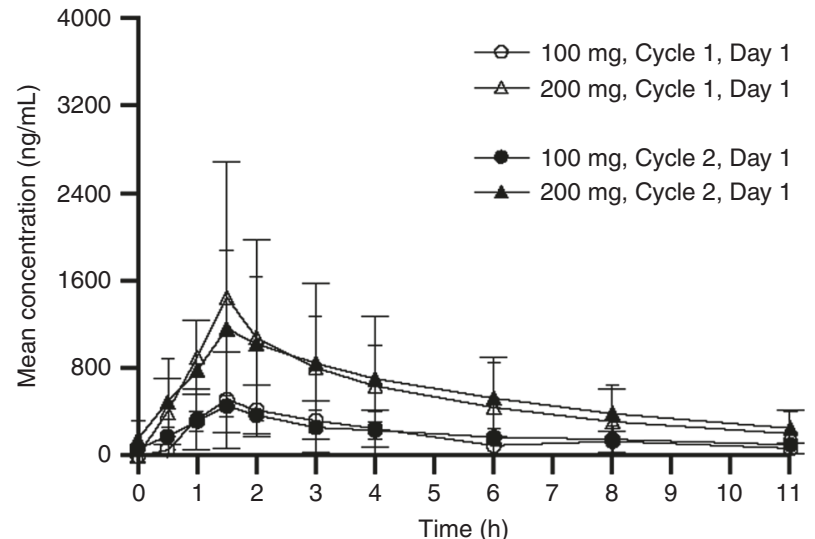

C

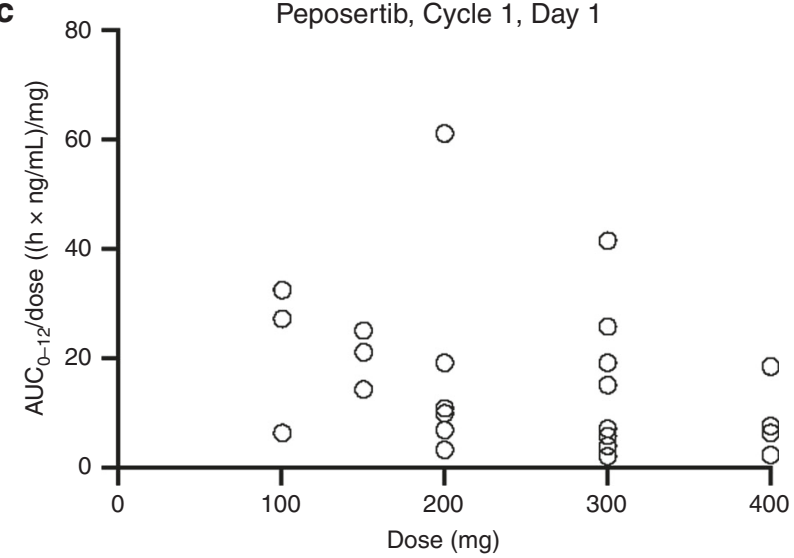

b

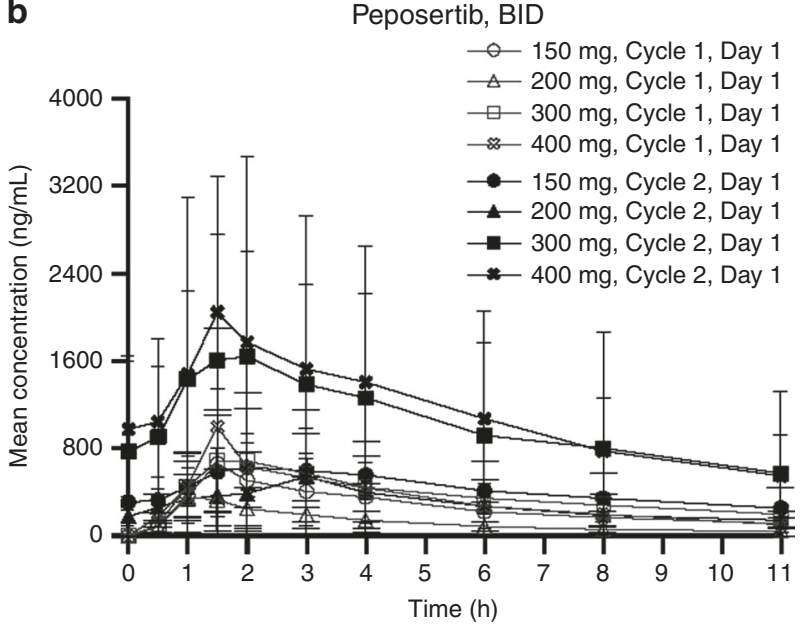

d

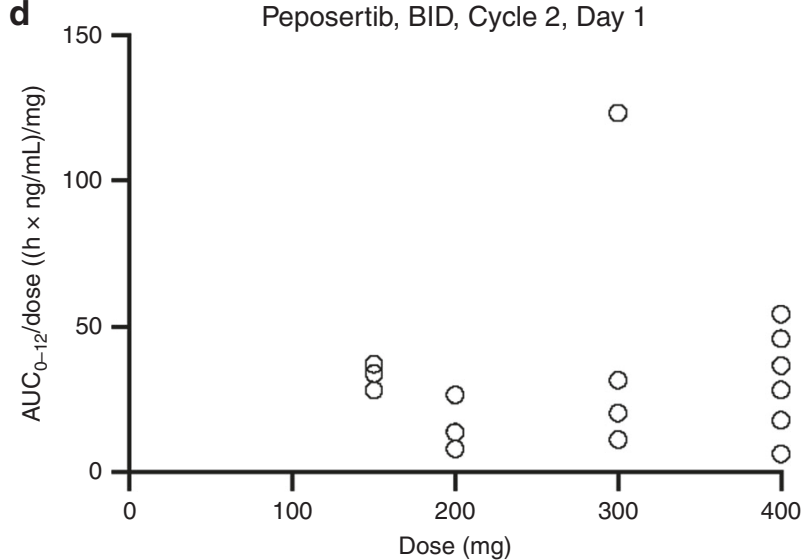

Fig. 2 Peposertib (formerly M3814) pharmacokinetics. Plasma concentration-time profiles of peposertib (formerly M3814) following a QD (PK Analysis Set; $n=6$ ) and b BID (PK Analysis Set; $n=25$ [Cycle 1], $n=17$ [Cycle 2]) dosing (linear scale). Dose-normalised AUC $0-12$ for peposertib by dose on c Cycle 1 Day 1 (QD and BID; PK Analysis Set; $n=25$ ) and d Cycle 2 Day 1 (BID; PK Analysis Set; $n=17$ ). AUC $0-12$ area under the plasma concentration-time curve from 0 to $12 \mathrm{~h}$, BID twice daily, PK pharmacokinetics, QD once daily.

TEAEs occurred in four (13\%) patients, all at the highest dose level of $400 \mathrm{mg}$ BID. Peposertib-related serious TEAEs were maculopapular rash in two patients; pyrexia and maculopapular rash in one patient; and nausea and maculopapular rash in one patient. Three patients died due to disease progression. One of these patients in the $400 \mathrm{mg}$ BID cohort had a TEAE leading to death (Grade 5 general physical health deterioration considered unrelated to peposertib). None of the deaths were considered treatment-related (Supplementary Table S1).

In the aspirin-naïve cohort ( $400 \mathrm{mg}$ BID, RP2D) a very weak effect on platelet aggregation was observed, which was not considered a safety signal.

\section{Pharmacokinetics}

Following oral administration, peposertib was quickly absorbed into the systemic circulation with a median $t_{\max }$ of $1.1-2.5 \mathrm{~h}$. The exposure parameters of peposertib showed high inter-individual variability across all doses as indicated by moderate to high geometric mean percent coefficient of variation $(\% \mathrm{CV})$ values (15.5-117\% for $C_{\max }$ i $12.5-124 \%$ for $\mathrm{AUC}_{0-12}$ ). Minimal accumulation of peposertib after QD and BID dosing was observed on cycle 2 day 1 , following multiple dosing, in line with the observed mean elimination half-life of $\sim 5.5 \mathrm{~h}$ (Fig. 2 and Supplementary Table S2). Plasma exposure of peposertib increased with increasing doses. However, high inter-individual variability in dose-normalised exposure prevented conclusive estimation of dose-proportionality (Fig. 2 and Supplementary Table S2).

\section{Pharmacodynamics}

Overall, a consistent and marked dose-dependent decrease in the PD biomarker level ( $p$-DNA-PK/total DNA-PK ratio) was observed in PBMCs, as a surrogate of tumour tissue, at early time points ( 3 and $6 \mathrm{~h}$ ) after peposertib administration (Fig. 3). A typical PK profile was shown in one patient treated at $100 \mathrm{mg}$ (Fig. 3a). In addition, peposertib concentration-dependent p-DNA-PK inhibition could be clearly established (Fig. 3b) with half-maximal inhibition $\left(\mathrm{IC}_{50}\right)$ observed at peposertib concentrations of approximately $200 \mathrm{ng} /$ $\mathrm{mL}$ (95\% confidence intervals, 149-286). These results demonstrate that peposertib inhibits DNA-PK in a time- and concentration-dependent manner, providing evidence of target engagement at doses below the MTD.

\section{Efficacy}

No objective tumour responses (complete or partial) were reported. However, 12 patients (39\%) had a best overall response of stable disease, of whom seven experienced prolonged stable disease for $\geq 12$ weeks. Of note, one patient with a metastatic renal cell carcinoma who previously progressed on sunitinib, pazopanib, axitinib and everolimus, and another patient with a tonsillar squamous cell carcinoma who previously progressed on prior cisplatin-based chemotherapy at the maximum administered dose, $400 \mathrm{mg}$ BID, remained on treatment for 36 weeks. Remarkably, a patient with a metastatic hepatocellular carcinoma and prior progression on sorafenib had stable disease for 69 weeks at the time of data cut-off. Five 
a
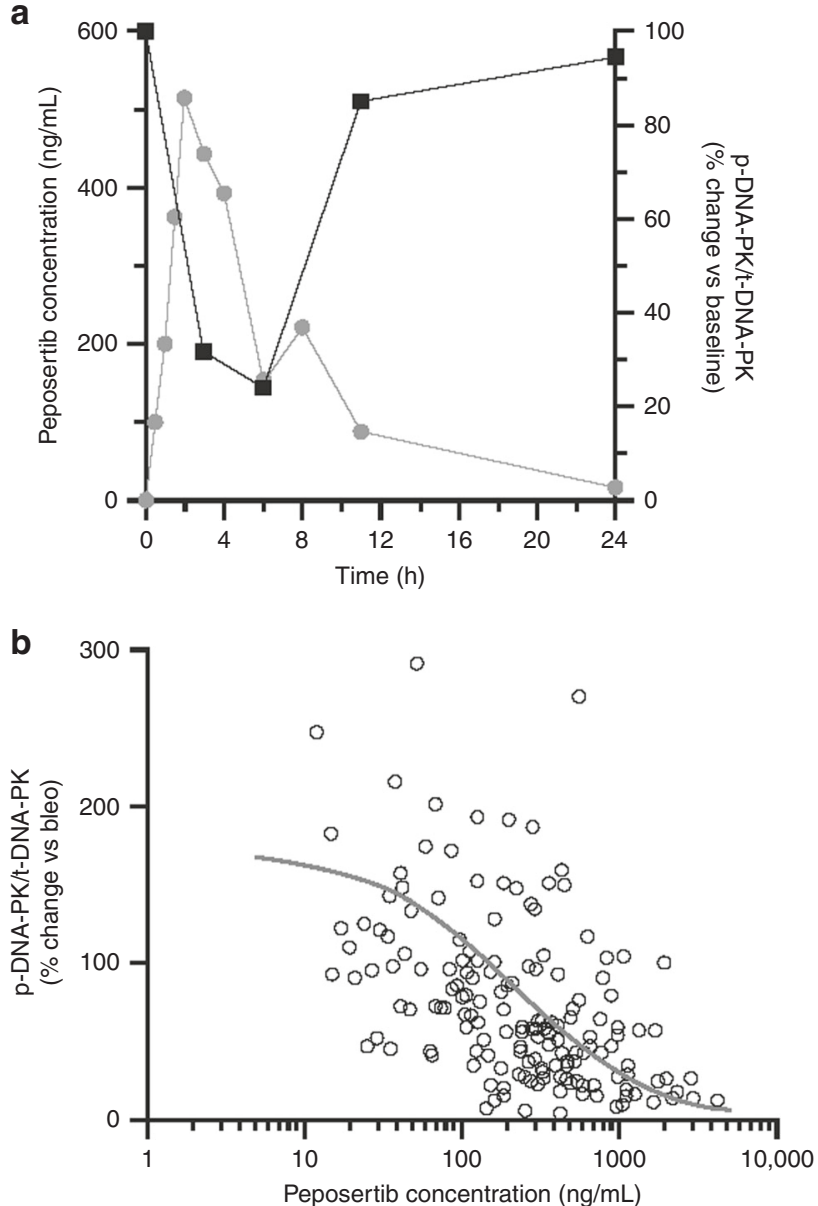

Fig. 3 DNA-PK inhibition by peposertib (formerly M3814). In a typical time-course profiles of PK (grey circles) and DNA-PK inhibition (black squares) after peposertib treatment from one patient treated with peposertib $100 \mathrm{mg}$ QD. DNA-PK inhibition on treatment is expressed as percentage change of $p-D N A-P K / t-D N A-$ PK from the pre-treatment value (time $h=0$ ). In $\mathbf{b}$ concentrationdependent inhibition of DNA-PK in response to peposertib treatment. Experimental concentration-response data are shown (open circles) and the line represents the $E_{\max }$ model fit to the data. Bleo bleomycin, DNA-PK DNA-dependent protein kinase, $E_{\max }$ maximum response, $\mathrm{p}$-DNA-PK autophosphorylated form of DNAPK on Ser ${ }^{2056}$, PK pharmacokinetics, QD once daily, t-DNA-PK total DNA-PK.

patients (16\%) were not evaluable (tumour assessment at screening only) (Fig. 4).

\section{DISCUSSION}

Here, we report the first-in-human study evaluating a DNA-PK inhibitor in patients with advanced cancer. Single-agent peposertib, a potent and selective inhibitor of the DNA-PK catalytic activity, $^{6-10}$ was well-tolerated when given orally QD or BID to patients with advanced solid tumours. The maximum administered dose was $400 \mathrm{mg} \mathrm{BID}$, and this was also declared the RP2D in monotherapy, based on the achieved predicted exposure -efficacy relationship, and evidence of target engagement. It was however decided not to conduct the dose-expansion part of the study as it was considered that the early efficacy signals were modest. This represents a limitation of the study because no early signs of efficacy could be ascertained in a tumour-specific or in a biomarker-selected patient population. From a clinical safety perspective, this study did not reveal any unexpected safety

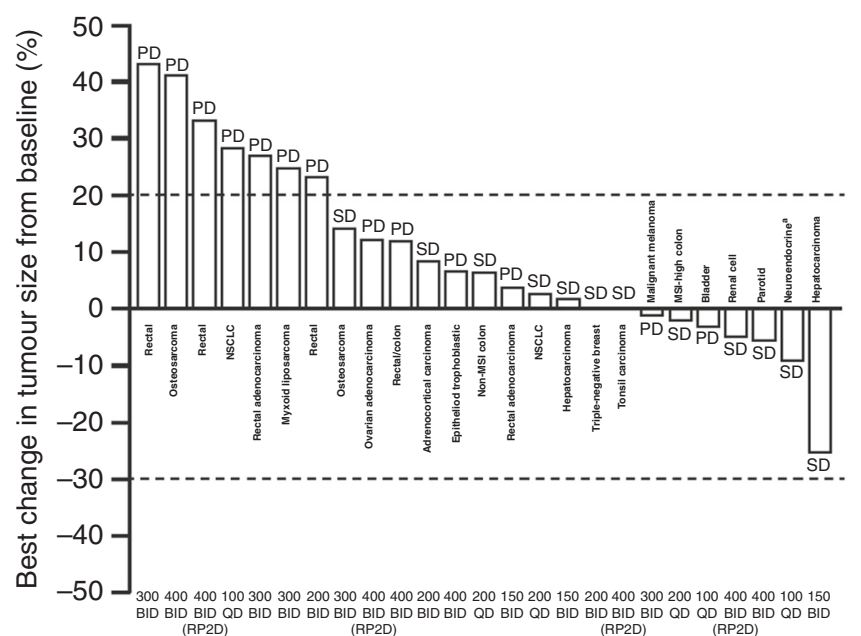

Fig. 4 Waterfall plot showing best percentage change in tumour size from baseline and best overall response. Peposertib (formerly M3814) dose (mg)/frequency of administration is shown for each patient on the $x$ axis. Dashed lines show the 20\% threshold for PD and the $30 \%$ threshold for partial response. ${ }^{a}$ Colon. BID twice daily, MSI microsatellite instability, NSCLC non-small cell lung cancer, PD progressive disease, QD once daily, RP2D recommended phase 2 dose, SD stable disease.

signals, in line with the preclinical toxicology data available at the time of study initiation. The only DLT reported consisted of a combination of low-grade, non-serious, mostly gastrointestinal AEs in one patient treated with $300 \mathrm{mg}$ peposertib BID.

Peposertib exhibits a high degree of selectivity when tested using a broad panel of 326 serine/threonine, tyrosine and lipid kinases. PI3K kinases, ATM, ATR, mammalian target of rapamycin and DNA-PK, are all members of the PI3K-related kinase family, which is characterised by high similarity in the kinase domain. Peposertib inhibited PI3K lipid kinases with significantly lower potency as demonstrated in preclinical studies. ${ }^{8}$ The inhibition of PI3K has been associated with some notable clinical AEs, such as hyperglycaemia, diarrhoea or rash. ${ }^{15}$ In this study, maculopapular rash was also reported in four patients. This was an intriguing clinical finding, as the preclinical experiments with peposertib showed only moderate inhibition of the phosphorylation of AKT, which is consistent with its weak inhibitory activity on the PI3K isoforms. ${ }^{8}$ Further data will be required to confirm any association between the occurrence of rash and the degree of PI3K inhibition elicited by peposertib in the clinical setting.

The present study also included a PD assessment of target engagement by peposertib in the clinical setting. Preclinical studies have demonstrated the feasibility of measuring DNA-PK inhibition by peposertib in human PBMCs, as an ex vivo surrogate of tumour tissue. The PD results of this study showed a concentration-dependent suppression of the phosphorylated fraction of DNA-PK indicating effective target inhibition by peposertib. This conclusion is limited to peripheral PBMCs since target engagement in paired-tumour biopsies were not collected.

To date, the use of DNA-PK inhibitors in clinical studies has been limited by the bioavailability of currently available molecules. ${ }^{16}$ Here, we show that the peposertib powder-in-capsule formulation was quickly absorbed into the systemic circulation, with slight accumulation observed with multiple QD and BID dosing. Peposertib plasma exposure increased with increasing dose, but dose proportionality could not be evaluated due to the large inter-patient variability in exposure. In addition, peposertib showed only a weak effect on platelet aggregation and this was not considered to be a safety signal. These findings do not support the exclusion of patients taking oral anti-coagulation medication from receiving peposertib treatment. 
The lack of partial responses with peposertib monotherapy according to RECIST (v1.1) is not entirely unexpected. The current study was performed in unselected patients, in whom the genetic background of the tumours evaluated was unknown; therefore, mechanisms of synthetic lethality associated with DNA-PK inhibition could not be exploited. Due to the lack of objective efficacy results, with no patients achieving a best response of partial or complete response, the dose-expansion cohort of this study which would have included genetic and molecular screening was not initiated. However, preclinical reports suggest that certain molecular aberrations in tumours, such as ATM loss, may lead to increased sensitivity to DNA-PK inhibition. ${ }^{17,18}$ In addition, peposertib has been shown to inhibit DNA DSB repair and enhance existing sensitivity to radiation in cancer cells through inhibition of DNA-PK kinase activity. ${ }^{6-9}$ Preclinical results indicate that combinations of peposertib with DNA DSB-inducing agents such as radiotherapy and certain chemotherapeutic agents (e.g. topoisomerase 2 inhibitors) are strongly synergistic and more likely to yield tumour responses. ${ }^{6,8,10}$ To this end, there are currently two ongoing clinical trials evaluating peposertib in combination with (chemo-) radiotherapy. A phase $1 \mathrm{a} / 1 \mathrm{~b}$ study is testing the administration of peposertib given with palliative radiation in metastatic solid tumours, as well as the combination of peposertib with high-dose cisplatin and high-dose radiation in locally advanced squamous cell carcinoma of the head and neck (NCT02516813). The second study is a phase $1 \mathrm{~b} / 2$ study evaluating the combination of peposertib with capecitabine and radiation for the neoadjuvant treatment of locally advanced rectal cancer (NCT03770689).

In conclusion, this first-in-human study of peposertib provides clinical evidence that peposertib inhibits DNA-PK activity and is well-tolerated when given orally as a single agent in doses up to $400 \mathrm{mg}$ BID. Currently ongoing studies in combination with chemo-radiation should shed light as to whether the addition of peposertib could enhance the efficacy of standard therapeutic regimens in selected tumour types, and therefore having the potential to become a new therapeutic option for patients with cancer.

\section{ACKNOWLEDGEMENTS}

The authors would like to thank patients, investigators, co-investigators, and the study teams at each of the participating centres and at Merck KGaA, Darmstadt, Germany. Laura Sponton, Ph.D., an employee of Merck KGaA, Darmstadt, Germany, provided support with developing and validating the assay used to measure the PD biomarker level, measuring clinical samples, and generating and interpreting PD data. Medical writing assistance was provided by Lisa Jolly, Ph.D., Jen Lewis, Ph.D. and Alexander T. Hardy, Ph.D. of Bioscript Science, Macclesfield, UK and funded by Merck KGaA, Darmstadt, Germany. The trial was sponsored by Merck KGaA, Darmstadt, Germany. This manuscript represents original work, which has not been previously published (in full) or simultaneously submitted for publication elsewhere; partial presentations took place at the American Society for Clinical Oncology Annual Meeting in $2017^{11}$ and at the European Society for Clinical Oncology Annual Meeting in $2018 .^{12}$

\section{AUTHOR CONTRIBUTIONS}

Study concept: K.B., L.D., B.S. Study design: K.B., L.D., B.S., J.H.M.S., M.J.A.d.J., M.M.-S. Data acquisition: M.T.J.v.B., P.S., A.A., H.M.W.V., J.H.M.S., M.J.A.d.J., D.N., L.D., M.M.-S. Quality control of data and algorithms: B.S. Data analysis and interpretation: S.E.B., M. K., K.B., I.D.-P., B.S., M.T.J.v.B., P.S., A.A., J.H.M.S., M.J.A.d.J., L.D. Statistical analysis: B.S. Manuscript preparation: M.K., I.D.-P., P.S., A.A., J.H.M.S. Manuscript editing: S.E.B., M.K., I.D.-P., M.T.J.v.B., P.S., A.A., H.M.W.V., J.H.M.S., D.N., L.S., M.M.-S. Manuscript review: S.E. B., M.K., K.B., I.D.-P., B.S., M.T.J.v.B., P.S., A.A., H.M.W.V., J.H.M.S., M.J.A.d.J., D.N., L.D., M. M.-S.

\section{ADDITIONAL INFORMATION}

Ethics approval and consent to participate The study was conducted in accordance with the ethical principles of the International Council for Harmonisation guideline for Good Clinical Practice and the Declaration of Helsinki, as well as with applicable local regulations. All patients provided written informed consent. The study sites received ethical approval from the following institutional review boards/ independent ethical committees: NVK for the region of Hovedstaden, Foundation BEBO, Jules Bordet Institute Ethics Committee, and the Ethics Committee Research UZ/KU Leuven.

\section{Consent to publish Not applicable.}

Data availability Any requests for data by qualified scientific and medical researchers for legitimate research purposes will be subject to the Merck KGaA, Darmstadt, Germany Data Sharing Policy. All requests should be submitted in writing to the Merck KGaA, Darmstadt, Germany data-sharing portal (https://www. merckgroup.com/en/research/our-approach-to-research-and-development/ healthcare/clinicaltrials/commitment-responsible-data-sharing.html). When Merck KGaA, Darmstadt, Germany has a co-research, co-development, or co-marketing or co-promotion agreement, or when the product has been out-licensed, the responsibility for disclosure might be dependent on the agreement between parties. Under these circumstances, Merck KGaA, Darmstadt, Germany, will endeavour to gain agreement to share data in response to requests.

Competing interests M.T.J.v.B., M.M.-S. and D.N. declare no potential conflicts of interest. A.A. has undertaken advisory roles and received speaker fees from Amgen, AstraZeneca, Bayer, Bristol-Myers Squibb, Eisai, Eli Lilly, Genomic Health, Hoffmann-La Roche, Ipsen, Leo Pharma, Merck \& Co., Novartis and Pfizer. M.J.A.d.J. has participated in advisory boards on behalf of Faron Pharmaceuticals. P.S. has received honoraria and has undertaken consultancy work for Deciphera. H.M.W.V. has participated in advisory boards on behalf of Lava Therapeutics and Glycostem, for which fees were paid to his institution. B.S., K.B., S.E.B. and M.K. are employees of Merck KGaA, Darmstadt, Germany. L.D. was an employee of Merck KGaA, Darmstadt, Germany, at the time of study conduct, and is currently an employee of Debiopharm International S.A., Lausanne, Switzerland. I.D.-P. is an employee of Ares Trading S.A., Eysins, Switzerland; an affiliate of Merck KGaA, Darmstadt, Germany. J.H.M.S. is patent holder on development of oral taxanes and shareholder of Modra Pharmaceuticals B.V.

Funding information Financial support for this study was provided by Merck KGaA, Darmstadt, Germany.

Supplementary information is available for this paper at https://doi.org/10.1038/ s41416-020-01151-6.

Publisher's note Springer Nature remains neutral with regard to jurisdictional claims in published maps and institutional affiliations.

\section{REFERENCES}

1. O'Connor, M. J. Targeting the DNA damage response in cancer. Mol. Cell 60, 547-560 (2015).

2. Hanahan, D. \& Weinberg, R. A. Hallmarks of cancer: the next generation. Cell 144, 646-674 (2011).

3. Desai, A., Yan, Y. \& Gerson, S. L. Advances in therapeutic targeting of the DNA damage response in cancer. DNA Repair 66-67, 24-29 (2018).

4. Davis, A. J., Chen, B. P. \& Chen, D. J. DNA-PK: a dynamic enzyme in a versatile DSB repair pathway. DNA Repair 17, 21-29 (2014).

5. Salles, B., Calsou, P., Frit, P. \& Muller, C. The DNA repair complex DNA-PK, a pharmacological target in cancer chemotherapy and radiotherapy. Pathol. Biol. 54, 185-193 (2006).

6. Fuchss, T., Mederski, W. W., Emde, U., Buchstallter, H.-P., Zenke, F., Zimmermann, A. et al. Highly potent and selective DNA-PK inhibitor M3814 with sustainable anti-tumor activity in combination with radiotherapy. Cancer Res. 77(13 Suppl), Abstract 4198 (2017).

7. Zenke, F. T., Zimmermann, A., Sirrenberg, C., Dahmen, H., Vassilev, L. T., Pehl, U. et al. M3814, a novel investigational DNA-PK inhibitor: enhancing the effect of fractionated radiotherapy leading to complete regression of tumors in mice. Cancer Res. 76(14 Suppl), Abstract 1658 (2016).

8. Zenke, F., Zimmermann, A., Sirrenberg, C., Dahmen, H., Kirkin, V., Pehl, U. et al. Pharmacological inhibitor of DNA-PK, M3814, potentiates radiotherapy and regresses human tumors in mouse models. Mol. Cancer Ther. 19, 1091-1101 (2020).

9. Klein, C., Dokic, I., Mairani, A., Mein, S., Brons, S., Haring, P. et al. Overcoming hypoxia-induced tumor radioresistance in non-small cell lung cancer by targeting DNA-dependent protein kinase in combination with carbon ion irradiation. Radiat. Oncol. 12, 208 (2017). 
A first-in-man phase 1 study of the DNA-dependent protein kinase... MT. J. van Bussel et al.

10. Sirrenberg, C., Zimmermann, A., Grombacher, T., Vassilev, L. T., Damstrup, L. Zenke, F. T. et al. A novel selective DNA-PK inhibitor, M3814, as a potential combination partner of Etoposide and Cisplatin in the therapy of lung cancer. Cancer Res. 77(13 Suppl), Abstract 4183 (2017).

11. van Bussel, M., Mau-Soerensen, M., Damstrup, L., Nielsen, D., Verheul, H. M. W. Aftimos, P. G. et al. A multicenter phase I trial of the DNA-dependent protein kinase (DNA-PK) inhibitor M3814 in patients with solid tumors. J. Clin. Oncol. 35 (15 Suppl), Abstract 2556 (2017).

12. Mau-Sorensen, M., van Bussel, M., Kuipers, M., Nielsen, D. L., Verheul, H. M., Aftimos, P. et al. Safety, clinical activity and pharmacological biomarker evaluation of the DNA-dependent protein kinase (DNA-PK) inhibitor M3814: results from two phase I trials. Ann. Oncol. 29(Suppl 8), viii654 (2018). (abstract 1845P).

13. Eisenhauer, E. A., Therasse, P., Bogaerts, J., Schwartz, L. H., Sargent, D., Ford, R et al. New response evaluation criteria in solid tumours: revised RECIST guideline (version 1.1). Eur. J. Cancer 45, 228-247 (2009).

14. National Institutes of Health. National Cancer Institute Common Terminology Criteria for Adverse Events (CTCAE) v4.03. https://evs.nci.nih.gov/ftp1/CTCAE/ CTCAE_4.03/CTCAE_4.03_2010-06-14_QuickReference_8.5x11.pdf (2010).

15. André, F., Ciruelos, E., Rubovszky, G., Campone, M., Loibl, S., Rugo, H. S. et al. Alpelisib for PIK3CA-mutated, hormone receptor-positive advanced breast cancer. N. Engl. J. Med. 380, 1929-1940 (2019).

16. Davidson, D., Amrein, L., Panasci, L. \& Aloyz, R. Small molecules, inhibitors of DNAPK, targeting DNA repair, and beyond. Front. Pharm. 4, 5 (2013).
17. Riabinska, A., Daheim, M., Herter-Sprie, G. S., Winkler, J., Fritz, C., Hallek, M. et al. Therapeutic targeting of a robust non-oncogene addiction to PRKDC in ATMdefective tumors. Sci. Transl. Med. 5, 189ra178 (2013).

18. Fok, J. H. L., Ramos-Montoya, A., Vazquez-Chantada, M., Wijnhoven, P. W. G., Follia, V., James, N. et al. AZD7648 is a potent and selective DNA-PK inhibitor that enhances radiation, chemotherapy and olaparib activity. Nat. Commun. 10, 5065 (2019).

Open Access This article is licensed under a Creative Common Attribution 4.0 International License, which permits use, sharing, adaptation, distribution and reproduction in any medium or format, as long as you give appropriate credit to the original author(s) and the source, provide a link to the Creative Commons license, and indicate if changes were made. The images or other third party material in this article are included in the article's Creative Commons license, unless indicated otherwise in a credit line to the material. If material is not included in the article's Creative Commons license and your intended use is not permitted by statutory regulation or exceeds the permitted use, you will need to obtain permission directly from the copyright holder. To view a copy of this license, visit http://creativecommons. org/licenses/by/4.0/.

(c) The Author(s) 2020 